\title{
Cognitive heterogeneity among community dwelling older adults with cerebral small vessel disease
}

\section{Authors:}

Ayan K. Dey ${ }^{1,2}$, Vessela Stamenova², Agnes Bacopulos ${ }^{2}$, Nivethika Jeyakumar ${ }^{2}$, Gary Turner ${ }^{3}$, Sandra E. Black $^{1,2,4,5}$, and Brian Levine , $^{1,2,4}$.

Note: Submitted on Feb. 19 ${ }^{\text {th }}$ 2018. Accepted Dec. $26^{\text {th }}$, 2018 to Neurobiology of Aging. In Press.

\section{Affiliations:}

1. Faculty of Medicine, Institute of Medical Science, University of Toronto, Toronto, Ontario, Canada

2. Rotman Research Institute, Baycrest Hospital, Toronto, Ontario, Canada

3. Department of Psychology, Faculty of Health, York University, Toronto, Ontario, Canada

4. Division of Neurology, Department of Medicine, University of Toronto, Ontario, Canada

5. L.C Campbell Cognitive Neurology Research Unit, Hurvitz Brain Sciences Research Program. Sunnybrook Health Sciences Centre, Toronto, Ontario, Canada

6. Department of Psychology, University of Toronto, Ontario, Canada

Keywords:

Cerebral small vessel disease; Executive function; Subjective cognitive complaints; White matter hyperintensities; Vascular cognitive impairment; Cognitive resilience

\section{Abbreviations:}

CSVD = Cerebral Small Vessel Disease, Non-CSVD = Healthy age-matched controls, WMHs $=$ White Matter Hyperintensities, CSF = cerebrospinal fluid, total supratentorial intracranial volume (STTIV), EF = Executive function component score, $\mathrm{SC}=$ Subjective complaint component score, $\mathrm{PS}=$ Processing speed component score, "-H" = high performing, "-L" = poor performing, CFQ = Cognitive Failures Questionnaire, DEX = Dysexecutive questionnaire.

\section{Highlights}

1. $50 \%$ of older adults in a case-control study with vascular risk factors had CSVD

2. Subjective complaints but not performance-based measures differentiated CSVD from controls.

3. In CSVD, preserved executive function appeared to come at the cost of reduced processing speed.

4. CSVD is associated with higher incidence of depressive symptoms.

\section{Corresponding Author:}

Ayan K. Dey, MD. Ph.D Candidate

Ayan.dey@mail.utoronto.ca

The Rotman Research Institute, 3560 Bathurst Street, $9^{\text {th }}$ Floor

Toronto, Ontario, M6A2E1 


\begin{abstract}
Some degree of ischemic injury to white matter tracts occurs naturally with age and is visible on magnetic resonance imaging as focal or confluent white matter hyperintensities (WMHs). Its relationship to cognition, however, remains unclear. To explore this, community-dwelling adults between the ages 55-80 years old completed structural imaging, neuropsychological testing, and questionnaires to provide objective measures and subjective experience of executive functioning. Volumetric lesion burden derived from structural MRI identified those with significant WMH burden $\left(\sim 10 \mathrm{~cm}^{3}\right)$. Half of those recruited met this criterion and were designated as the cerebral small vessel disease (CSVD) group. Subjective complaints but not objective test scores differentiated adults with and without CSVD. Hierarchical clustering revealed two CSVD subgroups that differentiated those with impaired versus preserved executive function relative to controls. Overall these results provide some explanation for behavioural heterogeneity often observed in studies of age-related white matter changes. They also support the use of questionnaires to assess subjective complaints that may be able to detect subtle effects of pathology not evident on standardized cognitive scores.
\end{abstract}

Word Count:171 


\subsection{Introduction}

First described by Otto Binswanger (1894), ischemic white matter lesions and small subcortical infarcts are commonly reported in older adults as incidental findings on MRI (Longstreth et al., 2002). Despite much research into the pathological mechanisms surrounding these lesions (Gouw et al., 2011; Rincon and Wright, 2014; Wardlaw et al., 2013a), little is known about their clinical significance and impact on function in the absence of vascular dementia. Even in the absence of trauma, autoimmune disease or cortical strokes, some degree of white matter damage occurs naturally with age -starting as early as the fifth decade of life (Wen et al., 2009). This is likely due to vascular pathology involving the deep penetrating end arteries of the brain that supply subcortical grey matter structures and white matter tracts, so called Cerebral Small Vessel Disease (Wardlaw et al., 2013b). These white matter lesions appear as hyperintensities on T2weighted magnetic resonance imaging and hence are often referred to as white matter hyperintensities (WMHs). The rate at which WMHs accumulate in older adults is accelerated in those with vascular risk factors (e.g., hypertension, smoking, dyslipidemia; (Khan et al., 2007; van Dijk et al., 2008). While extensive white matter hyperintensities are associated with vascular dementia (Jellinger, 2013), the relationship between mild to moderate white matter lesion burden and cognition is much less clear. For example, Burns and colleagues (2005) reported an association between deep and periventricular white matter lesion burden and reduced global cognition (including measures of visual memory, processing, and executive function) in those with dementia, but not in non-demented aging. Nevertheless, a recent meta-analysis has shown that individuals with cerebral small vessel disease (CSVD) without dementia, compared to healthy controls, demonstrate impairment predominantly in the domain of processing speed and to a slightly lesser extent executive function as measured through neuropsychological testing (Vasquez and Zakzanis, 2015). This relatively weaker relationship with executive function is surprising as executive dysfunction has long been considered a hallmark feature of vascular cognitive impairment (Prins et al., 2005; Sachdev et al., 2014). One possibility is that the weak relationship between $\mathrm{WMH}$ and behavior may reflect considerable heterogeneity within the population wherein some individuals are better able to compensate than others, but at the expense of processing speed. This tradeoff between accuracy and speed/efficiency is consistent with the temporal hypothesis for compensation (Martins et al., 2015) and the scaffolding theory of aging and cognition (Reuter-Lorenz and Park, 2014). Both theories posit a role for subjective 
complaints that may provide complementary information regarding cognition beyond what can be captured by traditional laboratory neuropsychological tests. For example, a mismatch between objective and subjective measures (e.g., high subjective complaints despite preserved performance), could suggest that performance is being boosted by compensatory processes at the cost of speed.

Another issue is that many studies exploring cognitive impairment in CSVD use a composite measure of executive function that linearly combine scores (with equal weight) from collections of diverse neuropsychological tests that were originally designed to assess different components of executive function (Lawrence et al., 2015). This is problematic because executive function is an umbrella term used to refer to a large collection of complex processes (Miyake et al., 2000) abilities that may not be equally affected by WMHs.

While addressing the limitations above, this investigation has two major aims. The first is to study the relationship between white matter lesion burden and objective and subjective measures of executive functioning in a sample of older, community dwelling adults with multiple vascular risk factors. The second aim is to identify the pattern of cognitive changes, whether observable or experienced, associated with CSVD. We hypothesize that in older, community dwelling adults there will be a significant negative relationship between processing speed (and to a lesser extent executive function) and WMH burden as well as a significant positive relationship between WMH burden and subjective complaints. Consistent with a compensation hypothesis (Martins et al., 2015), we predict that behavioural subgroups will emerge within the CSVD population, differentiating those with preserved executive function (relative to age-matched controls) but slower processing speed, and those with reduced executive functioning.

\subsection{Method}

\subsection{Inclusion/Exclusion Criteria}

Inclusion criteria consisted of age between 55-80 years old with at least one vascular risk factor from the following list: hypertension, hyperlipidemia, diabetes mellitus type 2, sleep apnea, active or history of cigarette smoking, history of transient ischemic attack, significant $1^{\text {st }}$ degree family history of cerebrovascular disease, or advanced age equal or greater than 70 years old. Risk factor information was recorded during screening and assessed based on self-report and/or prescription drug use (Dey et al., 2015). All participants were required to be fluent in written and 
spoken English. Aside from presence of CSVD-related lesions (WMHs and lacunes) on imaging, participants were otherwise healthy with no significant history of head trauma including concussion, cortical stroke, and/or other neurological/ psychiatric conditions that may unduly affect cognitive performance. As this study focuses on mild-moderate CSVD, any participants with a clinical diagnosis of dementia or meeting criteria for dementia were excluded regardless of white matter burden. Dementia was identified defined by self/other report of a pre-existing diagnosis and reported impaired activities of daily living. Other exclusion criteria included MRI contraindications, significant speech or language impairment (aphasia) and/or any significant motor or sensory deficits (e.g., uncorrected visual impairment) that may interfere with their ability to take part in the study. Pharmacological history was reviewed for each participant, two participants reported taking low-dose antidepressants (Duloxetine and Amitriptyline), and one participant reported a history of taking low-dose Clonazepam as needed for sleep (not taken during the period of this study).

\subsection{Participants \& Recruitment}

Between 2013 and 2017, older adults from the community meeting inclusion criteria were invited to participate in this prospective, population-based cohort study. Using previously reported mean effect sizes for executive function and processing speed in adults with vascular cognitive impairment relative to healthy controls (Vasquez and Zakzanis, 2015), we calculated an a-priori target sample size of 10-21 per group for a two-tailed hypothesis in order to achieve at least $80 \%$ statistical power. Participants were recruited through advertisements placed in the local newspaper, recruitment through the Rotman Research Institute research participant database, and through individuals who had volunteered for research at Cogniciti.com, a cognitive testing website affiliated with Baycrest. A total of 193 adults were screened for inclusion/exclusion criteria, of which 61 (31.6\%) were deemed eligible and invited to participate. Major reasons for exclusion included: lack of vascular risk factors (23), not meeting age cut-off (17), MRI contraindication (21), history of large vessel stroke (9), other neurological comorbidity (13) and significant history of recent or active psychiatric illness (9). Vascular risk factors were cross-checked against medications (Dey et al., 2015). Of those deemed eligible, 54 completed both cognitive assessment and structural imaging. After excluding individuals with incidental findings $(\mathrm{n}=7)$ and those with missing more than $10 \%$ of data fields for technical reasons or failure to complete the test battery, the final sample size was 46. Demographic and 
imaging characteristics of the population are described in Table 1. The mean age and years of education of our sample population were $70.13(\mathrm{SD}=5.08$ ) and 16.78 ( $\mathrm{SD}=3.31$ ), respectively. Participants reported $2.85(\mathrm{SD}=1.49)$ vascular risk factors on average. Relative to healthy controls those with CSVD were more likely to report experiencing subjective complaints (yes/no) $\left(\mathrm{X}^{2}=7.263, \mathrm{p}=0.007\right)$. Aside from this, the groups only differed with respect to variables relating to lesion burden that were used to determine group membership. That is, the two groups were similar with respect to demographic and physiologic variables such as age, education, number of vascular risk factors, handedness, and brain volume. Notably, the mean and median white matter hyperintensity volumes (adjusted for intracranial volume) in the CSVD group were $12.03 \mathrm{~cm}^{3}$ and $8.92 \mathrm{~cm}^{3}$, respectively. This falls within the range of "mild to moderate severity" identified in prior larger population studies (Rost et al., 2014; Zheng et al., 2012). Each participant gave informed consent to the protocol, which was approved by two separate Research Ethics Boards at Baycrest Hospital and Sunnybrook Health Sciences. Participants were not informed of the results of their neuroimaging assessment at the time of testing. Therefore, any effects on subjective or objective measures cannot be accounted for by demand characteristics related to participants' knowledge of their imaging results. All testing and scans were completed at Baycrest hospital. See Figure 1 for an overview of recruitment and study protocol. 


\section{Table 1: Summary of demographic and imaging characteristics across participants}

\begin{tabular}{|c|c|c|c|c|c|}
\hline \multirow[b]{2}{*}{ Age } & \multicolumn{2}{|c|}{$\begin{array}{c}\text { CSVD }(\mathrm{n}=23) \\
\text { Mean (SD), } \\
\text { Ratio or Count }(\%) \\
\end{array}$} & \multicolumn{2}{|c|}{$\begin{array}{c}\text { Non-CSVD }(\mathrm{n}=23) \\
\text { Mean }(\mathrm{SD}), \\
\text { Ratio or Count }(\%) \\
\end{array}$} & \multirow{2}{*}{$\begin{array}{c}\text { Sig. (2-tailed) } \\
0.359\end{array}$} \\
\hline & 71 & $(5.5)$ & 69.33 & $(4.64)$ & \\
\hline Years of Education & 16.45 & $(3.57)$ & 17.08 & $(3.11)$ & 0.661 \\
\hline Number of Vascular Risk Factors & 2.5 & $(1.65)$ & 2.17 & $(1.58)$ & 0.281 \\
\hline Hypertension & 13 & $(56.5 \%)$ & 14 & $(60.9 \%)$ & \\
\hline Diabetes Mellitus & 6 & $(26.1 \%)$ & 2 & $(8.7 \%)$ & \\
\hline Dyslipidemia & 13 & $(56.5 \%)$ & 9 & $(39.1 \%)$ & \\
\hline Cigarette Smoking (Current/Past) & 10 & $(43.5 \%)$ & 9 & $(39.1 \%)$ & \\
\hline Sleep Apnea & 3 & $(13.0 \%)$ & 1 & $(4.35 \%)$ & \\
\hline Family History of Cerebrovascular Disease & 5 & $(21.7 \%)$ & 9 & $(39.1 \%)$ & \\
\hline Prior Transient Ischemic Attack(s) & 6 & $(26.1 \%)$ & 5 & $(21.7 \%)$ & \\
\hline $\operatorname{Sex}(M: F)$ & & $: 12$ & 10 & & 0.79 \\
\hline Handedness (R: L) & & $8: 4$ & 21 & & 0.59 \\
\hline Subject Cognitive Complaints (Yes: No) & & $8: 5$ & 9: & & $0.007 *$ \\
\hline Supratentorial Total Intracranial Volume $\left(\mathrm{cm}^{3}\right)$ & 1249 & $(145)$ & 1203 & $(141)$ & 0.291 \\
\hline Grey Matter Volume $\left(\mathrm{cm}^{3}\right)$ & 565.1 & $(45.7)$ & 545.6 & $(53.3)$ & 0.193 \\
\hline White Matter Volume $\left(\mathrm{cm}^{3}\right)$ & 414.1 & $(59.2)$ & 399 & $(66.9)$ & 0.432 \\
\hline Sulcal Cerebrospinal Fluid $\left(\mathrm{cm}^{3}\right)$ & 220.8 & $(44.1)$ & 226.1 & $(45.1)$ & 0.698 \\
\hline Ventricular Cerebrospinal Fluid $\left(\mathrm{cm}^{3}\right)$ & 44.9 & $(22.5)$ & 32.5 & $(14.1)$ & $0.378+$ \\
\hline White Matter Hyperintensities $\left(\mathrm{cm}^{3}\right)^{*}$ & 11.267 & $(9.232)$ & 1.838 & $(1.573)$ & $<0.001 *$ \\
\hline Total Periventricular WMHs $\left(\mathrm{cm}^{3}\right)^{*}$ & 9.591 & $(8.830)$ & 1.630 & $(1.358)$ & $<0.001 *$ \\
\hline Total Deep WMHs $\left(\mathrm{cm}^{3}\right)^{*}$ & 1.675 & $(1.447)$ & 0.225 & $(0.271)$ & $<0.001 *$ \\
\hline Lacune Volume $\left(\mathrm{cm}^{3}\right)^{*}$ & 0.297 & $(0.363)$ & 0.0174 & $(0.019)$ & $<0.001 *$ \\
\hline Lacune Count* & 2.87 & $(1.89)$ & 0.45 & $(0.596)$ & $<0.001$ \\
\hline Adjusted White Matter Hyperintensities $\left(\mathrm{cm}^{3}\right)^{*+}$ & 12.031 & $(9.48)$ & 1.932 & $(1.515)$ & $<0.001 *$ \\
\hline $\begin{array}{l}\text { Percentage of White Matter affected by White } \\
\text { Matter Hyperintensity }(\%)\end{array}$ & 2.861 & $(2.43)$ & 0.45 & $(0.34)$ & $<0.001 *$ \\
\hline $\begin{array}{l}\text { Percentage of Supratentorial Intracranial Volume } \\
\text { affected by White Matter Hyperintensity (\%)* }\end{array}$ & 0.918 & $(0.73)$ & 0.149 & $(0.12)$ & $<0.001 *$ \\
\hline $\begin{array}{l}\text { Percentage of Supratentorial Intracranial Volume } \\
\text { affected by Lacunar volume }(\%)^{*}\end{array}$ & $2 \times 10^{-4}$ & $\left(3 \times 10^{-4}\right)$ & $1.4 \times 10^{-5}$ & $\left(2 \times 10^{-5}\right)$ & $<0.001 *$ \\
\hline
\end{tabular}

*differences remain significant at $\mathrm{p}<0.001$ after natural log transformation to adjust for skewness.

${ }^{+}$White matter hyperintensity volume corrected for total supratentorial intracranial volume.

SD: standard deviation.

\subsection{Imaging Parameters}

Imaging parameters were selected in accordance with the proposed acquisition standard for neuroimaging of small vessel disease described in the STRIVE working group position paper (Wardlaw et al., 2013b). Participants were scanned with a 3 Tesla magnetic resonance (MR) system (Siemens Magnetom Tim Trio system, Syngo MR 2006 VB17; Siemens, Germany) with 
a 12 channel matrix head coil. Anatomical scans were acquired via a T1-weighted threedimension (3D) magnetization-prepared rapid gradient-echo (MPRAGE) sequence (inversion time $[\mathrm{TI}]$ / repetition time $[\mathrm{TR}] /$ echo time $[\mathrm{TE}]=1100 / 2000 / 2.63 \mathrm{~ms}, 160$ slices, field of view $[$ FOV $]=256 \mathrm{~mm} \times 192 \mathrm{~mm}$, voxel size $=1 \times 1 \times 1 \mathrm{~mm}^{3}$, Flip angle $=9$ degrees, oblique axial slice orientation, 256x256 acquisition matrix, scan time $=6: 26)$. Proton density and T2-weighted images with a slice thickness of $3 \mathrm{~mm}$ were obtained using an interleaved axial multi-slice dual turbo spin echo sequence (TE1/TE2/TR = 19/93/2900ms, 48 slices, FOV = 224x185.5mm, $256 \times 256$ acquisition matrix, flip angle $=180$ degrees, scan time $=6: 24)$. For assessment of white matter lesion burden, a fluid attenuated inversion recovery (FLAIR) sequence was obtained $(\mathrm{TI} / \mathrm{TR} / \mathrm{TE}=2200 / 9000 / 108 \mathrm{~ms}, 48$ contiguous slices at $3 \mathrm{~mm}$ thickness, FOV $=224 \times 185.5 \mathrm{~mm}$, 256x256 acquisition matrix, flip angle $=165$ degrees, oblique axial slice orientation, scan time 7:41). This $\mathrm{T} 2$ sequence has the benefit of suppressing the hyperintense cerebrospinal fluid signal, allowing for visualization of lesions close to the ventricles. Total scan time for each participant was 56:06 minutes summed across all sequences, including diffusion scans, not discussed here.
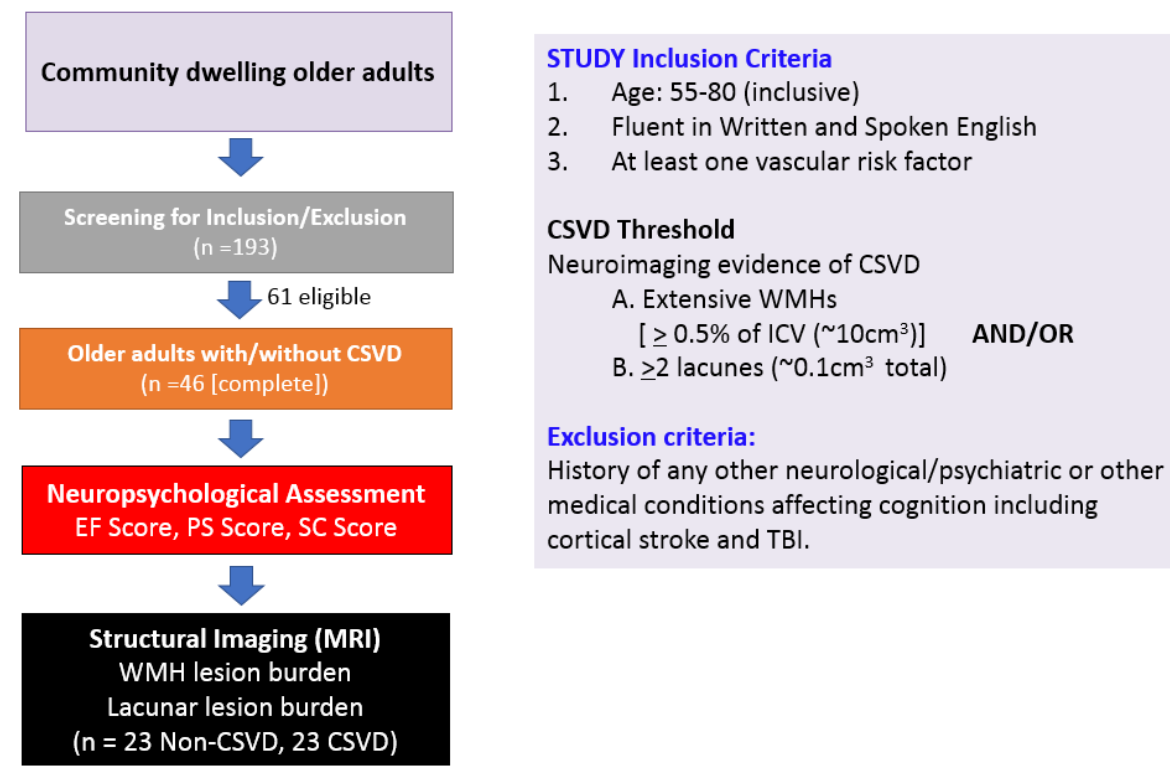

\section{Figure 1. Overview of recruitment and testing protocol}

193 older adults expressed interest in participating in the study. Following screening for inclusion/exclusion criteria (age 55-80 years old, English fluency, and at least one vascular risk factor), 61 were invited to participate in the study. 54 completed both cognitive assessment and structural imaging. However, eight were subsequently excluded due to incidental findings and missing data, leaving a final sample size of 46 . Ultimately, 
the participants were split equally among those with $(n=23)$ and without CSVD $(n=23)$ based on neuroimaging evidence of significant white matter lesion burden and/or the presence of 2 or more lacunes.

\subsection{Volumetric Quantification of Lesion Burden}

All scans were processed through a pipeline developed at Sunnybrook Health Sciences Centre (http://sabre.brainlab.ca/docs/processing/index.html). See Figure 2 for a visual summary of the processing pipeline. Image processing and tissue segmentation were performed using ITK-SNAP version 3.6 (Yushkevich et al., 2006). The first step was the co-registration of the PD and T2 images to T1 space. The next step (referred to as "Brain-Sizer") is described in detail in Ramirez et al. (2011) involved removing non-brain voxels. In this step, a brain-extraction algorithm (Kovacevic et al., 2002) was run on the PD/T2 images, which were further manually edited on the T1 images. Then, following image alignment, using the T1 images, the brain was segmented into four tissue classes: grey matter, white matter, sulcal, and ventricular cerebrospinal fluid (CSF). Segmentation was performed using a local and global histogram-based automatic segmentation tool (Kovacevic et al., 2002). Designation of the ventricles and removal of the cerebellum was then done manually on the T1 image. The next step was lesion segmentation, which was accomplished with a C-means algorithm called Fuzzy Lesion Extractor (FLEX) that was run on the FLAIR images (Gibson et al., 2010). All lesion segmented images were then

further manually edited by reassigning false negative and false positive voxels. An automated 3D connectivity operation further classified all subcortical hyperintensities into either periventricular or deep white matter hyperintensities. All voxel clusters that were connected in 3D to the ventricles were classified as periventricular WMHs, and the remaining WMH voxels were classified as deep white WMHs. WMHs were also classified as lacunar or non-lacunar (aka "white matter hyperintensity"). This classification was done by comparing how each identified WMH was classified on the T1-image: WMH voxels that were classified as CSF during the T1 tissue segmentation were classified as lacunar, while those classified as white matter were classified as non-lacunar. For counts, only lacuna with diameters between 3-15mm were included. The diameter was measured by counting the voxels as the scan resolution was 1x1x1mm. The segmented and processed image was processed using the Semi-Automated Brain Region Extraction (SABRE 2.0) method (Dade et al., 2004; Ramirez et al., 2011), which yields 26 regions customized to each participants' brain anatomy using 7 brain landmarks and coordinates and six sulcal tracings manually identified on each scan. The final output was a 
comprehensive volumetric profile of an individual's brain tissue volumes with regionalized segmentation data for grey matter, white matter, ventricular CSF, sulcal CSF, lacunar and nonlacunar periventricular WMH, lacunar and non-lacunar deep WMH in each of the 26 SABRE regions. In each participant, total supratentorial (i.e., cerebrum above the tentorium cerebelli) intracranial volume (ST-TIV) was calculated as the sum of all segmentation values while total WMH burden was calculated by summing periventricular WMH and deep WMH across the brain. To correct for headsize both total WMH burden and total lacunar volume measures were expressed as a percentage of ST-TIV. Percentage of white matter affected by WMH was calculated by dividing WMH burden by total intracranial white matter volume (i.e., deep WMH + periventricular $\mathrm{WMH}+$ normal appearing white matter). Because the positive tail of the distributions for WMH burden and lacunar burden were skewed, both variables were natural log transformed prior to statistical analysis.

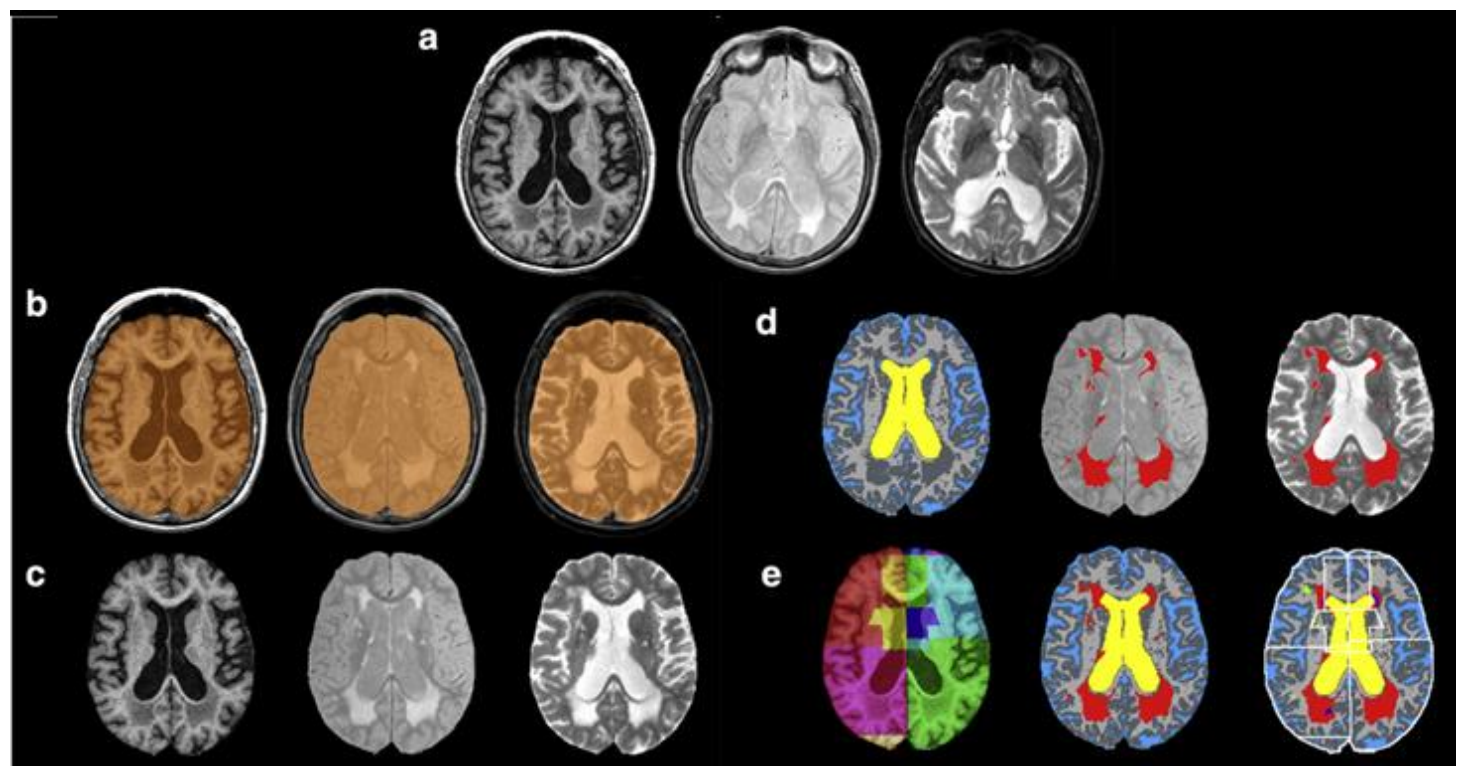

Figure 2. Processing Pipeline for Quantification of Lesion Burden

A) T1, PD, T2 and FLAIR MRI (not shown). B) Co-registration of PD, T2, and FLAIR to T1 space, with binary mask overlaid for brain extraction. C) Extraction of brain and sub-dural CSF for tissue segmentation preparation. D) T1-segementation (cerebrospinal fluid = blue, white matter $=$ light grey, grey matter $=$ dark grey) and lesion segmentation using the Fuzzy Lesion Extractor (FLEX) pipeline (red overlay on PD-T2 image). E) T1 segmentation corrected for subcortical hyperintensities and separated into SABRE regional compartments (colours present different regions). This figure has been reproduced and modified with permission from Ramirez et al. (2011). Details available at www.sabre.brainlab.ca 


\subsection{Threshold for Cerebral Small Vessel Disease}

While there is no widely held consensus on a precise diagnostic threshold of lesion burden for CSVD from quantified images, DeCarli and colleagues (1995) found that WMH burden exceeding $0.5 \%$ of intracranial volume $\left(\sim 10 \mathrm{~cm}^{3}\right)$ to be associated with larger ventricular volume, reduced brain volume and frontal glucose metabolism, and reduced executive function scores in a sample of healthy older adults. This approximates a score of 1-2 on the Fazekas scale (a widely used categorical measure for visual rating of WMH; (Fazekas et al., 1987) (Svärd et al., 2017; White et al., 2013). That said, we classified those with white matter hyperintensity volume exceeding $0.5 \%$ of intracranial volume $\left(\sim 10 \mathrm{~cm}^{3}\right)$ as having CSVD. A CSVD diagnosis was also assigned to participants with two or more small subcortical infarcts/lacunes (Wardlaw et al., 2013b) and/or one strategically placed subcortical infarct/lacune (within the thalamus, basal ganglia or internal capsule) as assessed by a neurologist or neuro-radiologist. Lacunes were defined as focal hyperintensities on T2-weighted images, $3 \mathrm{~mm}-15 \mathrm{~mm}$ in diameter, and with a corresponding prominent hypointensity on T1-weighted images (Wardlaw et al., 2013b). Distribution of lesion burden across groups is described in the scatterplot in Figure 3. Five participants were identified as meeting criteria for CSVD based on lacune count $(\geq 2)$ despite having sub-threshold levels of white-matter hyperintensity volume. No individuals were identified as having CSVD solely on the basis of a strategic lesion.

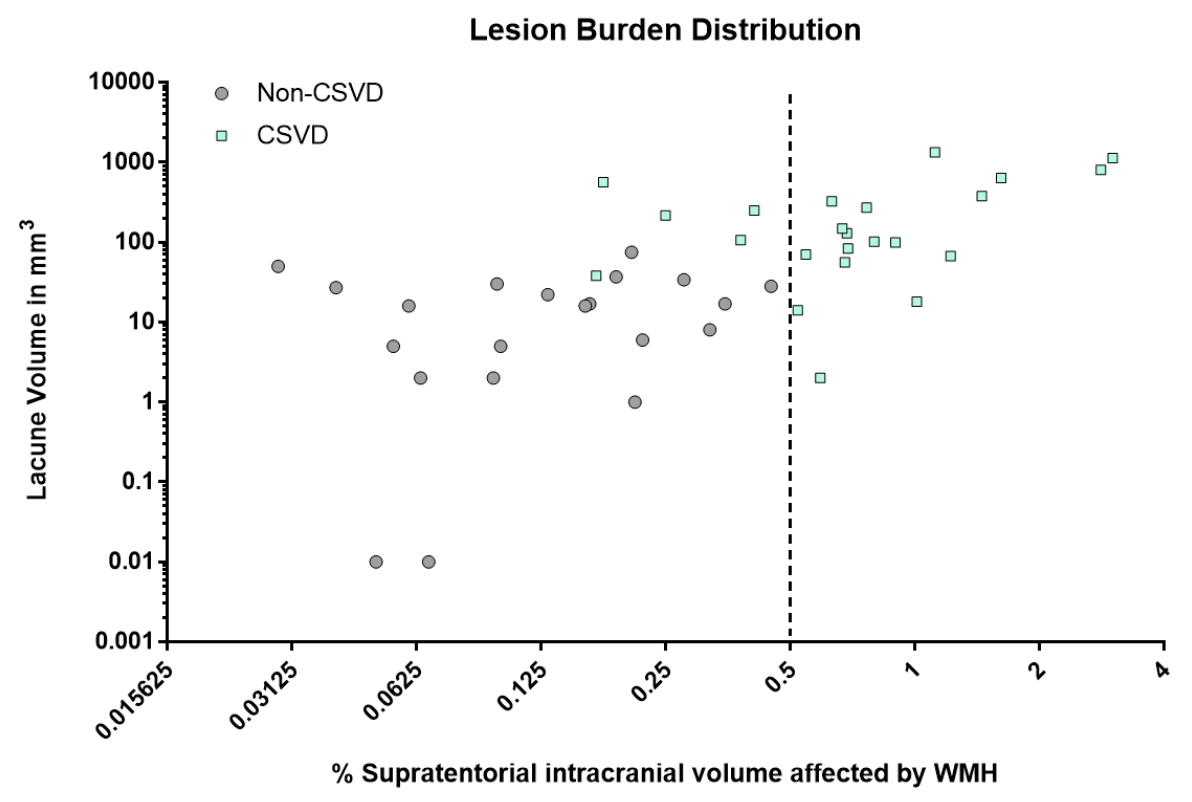

Figure 3. Distribution of lesion burden across sample 
This scatterplot displays the distribution of lesion burden across the sample, with CSVD represented as aqua squares and Non-CSVD controls represented by grey circles. White matter hyperintensity expressed as a percentage of supratentorial intracranial volume is plotted on the $\mathrm{X}$ axis, while lacune volume $\left(\mathrm{mm}^{3}\right)$ is plotted on the Y axis. Scales were adjusted in order to accommodate skewness of the lesion burden measures (log 10) The dotted line represents the thresholds used to identify the groups. 5 individuals met criteria for CSVD based on number of lacunes alone $(\geq 2)$.

\subsection{Cognitive Assessment}

All participants also completed a two-hour battery of neurocognitive tests and functional questionnaires specifically designed to assess attention and executive function. These tests include the Symbol-Digit Modalities Test (Smith, 1978), the Self-Ordered Pointing Test (Petrides and Milner, 1982), and Digit Span. Additionally, neuropsychological tests from the Delis-Kaplan Executive Function System were utilized, namely: the Trail-Making Test, the Tower Test, the Colour-Word Inference Test and tests of Verbal/Design/ Category Fluency (Delis et al., 2001; Spreen and Strauss, 1998). To increase sensitivity of behavioural assessment, functional-behavioural measures such as the Hotel task (Manly et al., 2002) (a task designed to assess an individual's ability to manage multiple goals and tasks) and the Test of Everyday Attention (visual elevator subtest) (Robertson et al., 1996) were included.

Subjective complaints were assessed through self and close-other ratings on the Cognitive Failures Questionnaire (CFQ) (Broadbent et al., 1982), and the Dysexecutive Questionnaire (DEX) (Wilson et al., 1996) - validated questionnaires designed to measure behavioural problems associated executive dysfunction in daily life such as poor attentiveness, memory failures, and disinhibition. On the CFQ participants were asked to answer 25 questions and rate their responses on a 5 point scale from "never" to "very often", with higher scores suggesting poor attentiveness and greater memory failures. Sample questions from the CFQ include: “do you fail to notice signposts on the road?" and "do you start doing one thing at home and get distracted into doing something else (unintentionally)?”. The DEX questionnaire contains 20 questions rated on a 5 point scale concerning inhibition, affect, planning, and memory. For example, "I have difficulty realizing the extent of my problems and am unrealistic about the future". The scores from these questionnaires were combined into a composite score for subjective complaints (SC). Subjective complaints were neither associated with executive function $(E F ; r=-0.12, p=0.432$ ) nor processing speed $(P S ; r=-0.23, p=0.124)$, consistent with the notion that these questionnaires reflect performance on unstructured daily life tasks that does 
not overlap with performance on structured neuropsychological tests (Burgess et al., 1998). It is also possible a decline in executive function may hinder insight into one's deficits (Brookes et al., 2014).

Finally, the Hopkins Verbal Learning Test-Revised (Benedict et al., 1998) was included in the battery in order to identify any individuals with significant impairments in learning and/or memory. While not an exclusion criterion, none of the participants included in the final sample were found to be impaired on the HVLT based on their normalized T-Score.

To reduce the test data according to its underlying structure, subjective and objective measures were separately entered into a principal component analysis (PCA) with varimax rotation and Kaiser Normalization. This was performed using the dimension reduction feature of SPSS version 24.0. Prior to entry into the principal component analysis, all timed variables were inversed and any scores that were significantly skewed were natural log transformed. From the PCA, only components with eigenvalues above 1 were selected. Among objective measures, two orthogonal components scores representing executive function/working memory (EF) and speed of processing (PS) were identified, accounting for $40.9 \%$ of the total variance. Among subjective measures, only one component (subjective complaints; SC) was identified, accounting for $65.6 \%$ of the total variance. Factor weights for the component scores are reported in the supplemental materials. Additionally, information regarding self-reported vascular risk factors (hypertension, diabetes mellitus, dyslipidemia, cigarette smoking, family history of cerebrovascular disease, sleep apnea, and history of transient ischemic attacks) and depressive symptoms as measured by the 30 question geriatric depression scale (GDS)(Yesavage et al., 1982) were also collected.

\subsection{Statistical Analysis}

All data analysis was performed with SPSS Statistics 24, and R Studio (version 1.0.143) was used primarily for visualization. Descriptive statistics for the data (e.g., quantitative imaging measures, cognitive measures, and demographic information) across the entire sample as well as between clinical groups (Non-CSVD vs. CSVD) are described in Table 1. For continuous variables, independent samples T-tests were used to assess group differences. Data for skewed variables such as lesion burden were natural log transformed before analysis. For categorical variables, chi-square test for independence (with Yates continuity correction) was used to compare group differences. 
To address the first aim of this study, multiple regression was used to assess the ability of lesion burden measures (white matter hyper-intensity volume and lacunar volume) to predict cognition (EF, PS, and SC score) in older adults across our entire sample after accounting for variance attributable to age and education. Separate regression models were run for each dependent cognitive measure. Both lesion burden measures were entered as a percentage of total supratentorial intracranial volume to correct for head size and natural log transformed to correct for skewness. Age and education were also added to the model. Preliminary analyses were conducted to ensure no violation of the assumptions of normality, linearity, multicollinearity, and homoscedasticity. Pearson correlations between the dependent and independent variables were also explored. Concerning the second aim of this study, to explore patterns of cognitive change associated with CSVD, a one-way multivariate between-groups analysis of covariance (MANCOVA) was conducted to compare the cognitive profile of older adults with and without CSVD across the three cognitive measures (EF, PS and SC). The clinical group was the sole fixed factor independent variable, while EF, PS and SC scores were dependent variables. Age and education were also entered as covariates in this analysis. Distribution of the dependent variables in both the groups was visualized using a kernel density plot. Finally, to investigate behavioural subgroups within the CSVD sample, hierarchical clustering was employed to identify data clusters with EF, PS and SC scores as inputs. Ward's method was selected as the clustering method and squared Euclidean distance as the unit of measure. This analysis identified two data clusters within the CSVD population which primarily differed with respect to their EF component score. Accordingly, these data clusters were labelled as CSVD-High performing (CSVD-H) and CSVD-Low performing (CSVD-L). Non-CSVD controls were not included in the cluster analysis as we were interested in exploring how these subgroups compare to healthy controls. That said, to assess between-cluster differences in demographic and quantitative imaging measures, a one-way ANOVA was performed, considering participant cluster as the independent variable and demographic/lesion burden measures (age, years of education, number of vascular risk factors, WMH lesion burden, lacune lesion burden) as dependent variables. A one-way MANCOVA was run to explore if differences between the CSVD clusters and controls across cognitive measures persist after adjusting for covariates. In this final analysis, simplified group/cluster membership (Non-CSVD vs. CSVD-H vs. CSVD-L) was entered as the independent variable, and the cognitive measures of EF, PS, and SC scores were entered as dependent variables. Age, education, sex, and WMH lesion burden were also entered as 
covariates. Follow-up Bonferonni-corrected pairwise comparisons were performed to explore the relative performance between age-matched controls the two CSVD subgroups.

\subsection{Results}

\subsection{Relationship between lesion burden and cognition}

Multiple regression was used to assess the ability of WMH and lacunar lesion burden to predict cognition (processing speed, executive function, and subjective complaints) while controlling for the influence of age, education and sex. Findings are summarized in Table 2. While none of the regressions models met criteria for significance, there was a significant positive correlation between subjective cognitive complaints and WMH burden $(\mathrm{r}=0.295, \mathrm{p}=0.023)$. WMH lesion burden, overall, was a relatively strong predictor of cognition across all the models. Although this should be interpreted with caution, this finding is consistent with larger studies (Molad et al., 2017; Uiterwijk et al., 2016).

Table 2: Multiple regression analysis - predictors of cognitive function.

\begin{tabular}{|c|c|c|c|c|c|c|c|}
\hline Dependent Variable & Model & R Square & Model Sig. & Beta & Sig. & $\begin{array}{c}\text { Pearson } \\
\text { Correlation }\end{array}$ & Sig. \\
\hline \multirow{5}{*}{ PS Score } & Age & \multirow{5}{*}{0.128} & \multirow{5}{*}{$\begin{array}{c}\mathrm{F}(5,40)=1.174 \\
\mathrm{p}=0.339\end{array}$} & -0.131 & 0.402 & -0.197 & 0.095 \\
\hline & Years of Education & & & 0.054 & 0.730 & 0.144 & 0.235 \\
\hline & Sex & & & -0.185 & 0.246 & -0.230 & 0.062 \\
\hline & WMH Lesion Burden ${ }^{+}$ & & & -0.253 & 0.224 & -0.230 & 0.062 \\
\hline & Lacunar Lesion Burden $^{+}$ & & & 0.081 & 0.694 & -0.058 & 0.351 \\
\hline \multirow{5}{*}{ EF Score } & Age & \multirow{5}{*}{0.092} & \multirow{5}{*}{$\begin{array}{c}\mathrm{F}(5,40)=0.808 \\
\mathrm{p}=0.551\end{array}$} & -0.108 & 0.498 & -0.137 & 0.183 \\
\hline & Years of Education & & & 0.049 & 0.757 & 0.025 & 0.436 \\
\hline & Sex & & & 0.172 & 0.292 & 0.122 & 0.209 \\
\hline & WMH Lesion Burden ${ }^{+}$ & & & -0.294 & 0.168 & -0.227 & 0.064 \\
\hline & Lacunar Lesion Burden ${ }^{+}$ & & & 0.138 & 0.511 & -0.087 & 0.284 \\
\hline \multirow{5}{*}{ SC Score } & Age & \multirow{5}{*}{0.171} & \multirow{5}{*}{$\begin{array}{c}\mathrm{F}(5,40)=1.655 \\
\mathrm{p}=0.168\end{array}$} & -0.252 & 0.101 & -0.181 & 0.114 \\
\hline & Years of Education & & & -0.097 & 0.525 & -0.178 & 0.119 \\
\hline & Sex & & & 0.104 & 0.501 & 0.104 & 0.247 \\
\hline & WMH Lesion Burden ${ }^{+}$ & & & 0.355 & 0.083 & 0.295 & $* 0.023$ \\
\hline & Lacunar Lesion Burden $^{+}$ & & & -0.039 & 0.847 & 0.185 & 0.109 \\
\hline
\end{tabular}

*Significant at $\mathrm{p}<0.05$.

${ }^{+}$Expressed as \% of supratentorial intracranial volume and natural log transformed

\subsection{Cerebral Small Vessel Disease and cognition}

Between-groups multivariate analysis of covariance (MANCOVA) revealed group differences across cognitive measures while adjusting for covariates (age and education). Subjective 
complaints $(\mathrm{F}[1,42]=7.865, \mathrm{p}=0.008$, partial eta squared $=0.1580)$ but not processing speed $(\mathrm{F}[1,42]=1.29, \mathrm{p}=0.263)$ or executive function $(\mathrm{F}[1,42]=0.35, \mathrm{p}=0.557)$ significantly distinguished older adults with and without CSVD. An independent-samples t-test showed no significant differences in subjective scores between males and females; $t(44)=0.691, p=0.493$. The two groups were also indistinguishable with respect to memory performance as assessed by the Hopkins Verbal Learning Test delayed recall $(F[1,42]=2.374, p=0.131)$ and recognition subtests $(F[1,42]=0.048, p=0.828)$. The groups, however, differed with respect to reporting of depressive symptoms as assessed by the Geriatric Depression Scale (long form; GDS), with the CSVD group reporting greater depressive symptoms $(\mathrm{F}[1,42]=5.063, \mathrm{p}=0.030$, partial eta squared $=0.118$ ). None of the participants met criteria for clinical major depression, and 39/46 $(85 \%)$ had GDS scores < 10 . These results are summarized in Table 3 with group differences in cognitive measures visualized in the dot plot in Figure 4a. Density plots highlighting the distribution of the data across groups are shown in Figure $4 \mathbf{b}$.

\section{Table 3: Cognition in older adults with and without CSVD}

\begin{tabular}{lccrr} 
Cognitive Measure & $\begin{array}{c}\text { Non-CSVD Mean } \\
(\mathbf{9 5 \%} \text { CI) }\end{array}$ & $\begin{array}{c}\text { CSVD Mean } \\
\text { (95\% CI) }\end{array}$ & $\begin{array}{c}\text { p- } \\
\text { value* }\end{array}$ & $\begin{array}{c}\text { Partial eta } \\
\text { Squared }\end{array}$ \\
\hline Neuropsych Component 1 (PS) & $0.168(-0.252$ to 0.588$)$ & $-0.168(-0.588$ to 0.252$)$ & 0.263 & 0.03 \\
Neuropsych Component 2 (EF) & $0.090(-0.342$ to 0.522$)$ & $-0.090(-0.522$ to 0.342$)$ & 0.557 & 0.008 \\
Subjective Complaints (SC) & $-0.386(-0.776$ to 0.004$)$ & $0.386(-0.004$ to 0.776$)$ & $\mathbf{0 . 0 0 8 *}$ & 0.158 \\
HVLT Delayed Recall & $10.373(9.602$ to 11.145$)$ & $9.540(8.768$ to 10.311$)$ & 0.131 & 0.053 \\
HVLT Recognition & $11.103(10.653$ to 11.554$)$ & $11.027(10.576$ to 11.478$)$ & 0.828 & 0.001 \\
Geriatric Depression Scale & $3.438(1.303$ to 5.572$)$ & $6.856(4.918$ to 8.794$)$ & $\mathbf{0 . 0 3 0 *}$ & 0.118
\end{tabular}




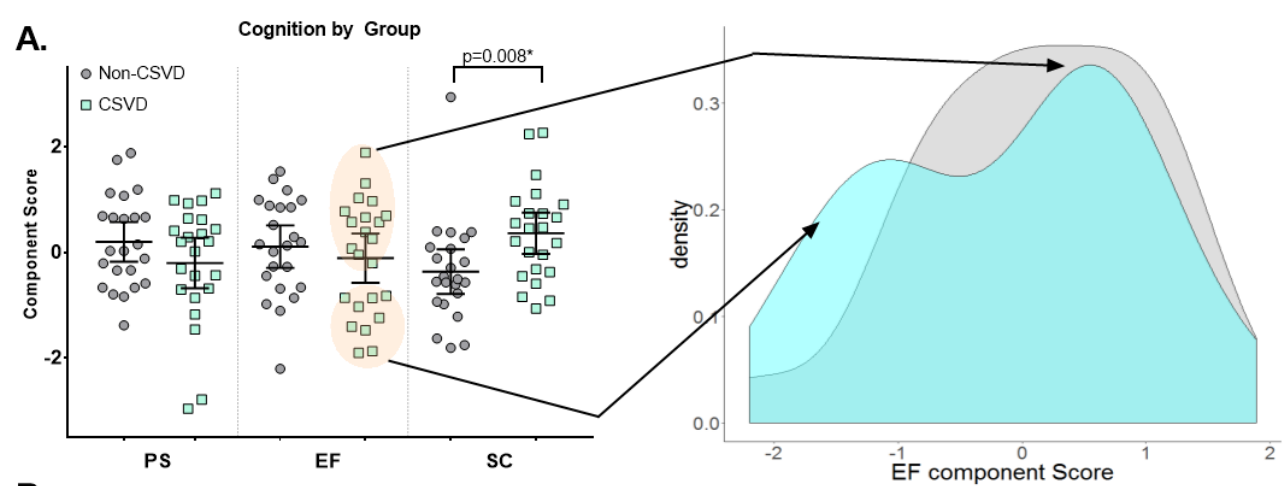

B.
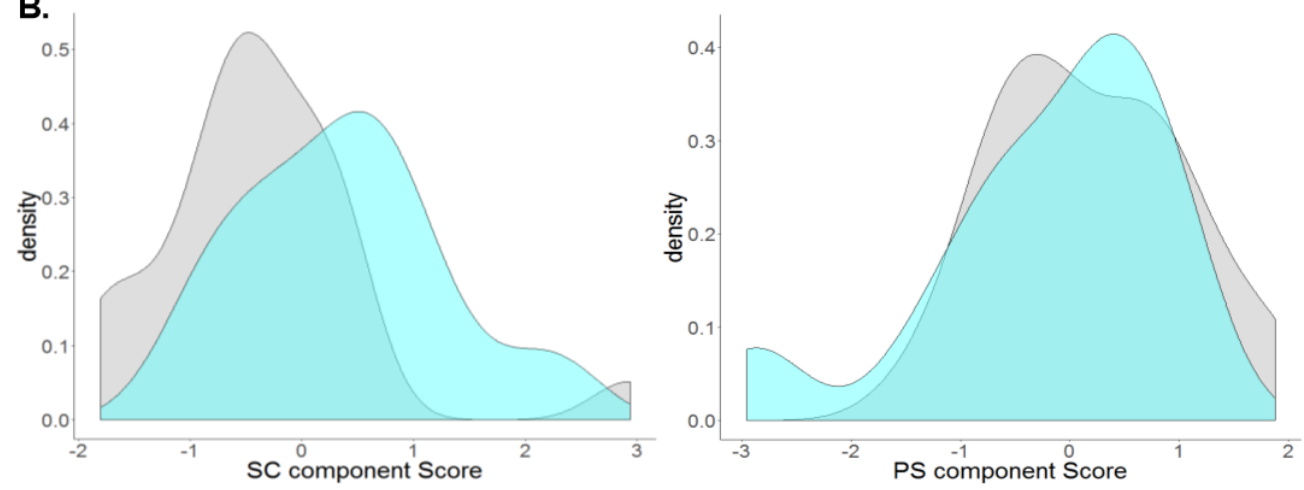

Figure 4. Density plots of cognition in older adults with and without CSVD.

A. Dot plot showing group differences across component scores derived from the neuropsychological assessment battery and questionnaires. The error bars represent $95 \%$ confidence intervals. From left to right; processing speed (PS), executive function (EF) and subjective complaints (SC). Of the measures, only subjective complaints significantly distinguished the two groups, with more complaints reported among those with CSVD $(\mathrm{p}=0.008)$. B. Density plots show the spread of data, with signs of a possible bimodal distribution visible on the plot for executive function (EF). Non-CSVD controls are represented in grey, while the CSVD group is represented in light blue.

\subsection{Behavioural subgroups}

Using EF, PS and SC component scores as inputs, hierarchical clustering identified two data clusters across the CSVD sample. The two CSVD subgroups were named CSVD-H (green) and CSVD-L (blue). "H" and "L" here represent high and low EF components scores, respectively. As summarized in Table 4, despite behavioural differences, one-way ANOVA found no statistically significant differences between the two clusters with respect to age, years of education, number of vascular risk factors, WMH lesion burden and number of lacunes. One notable difference is that females represent a larger proportion of those in the higher performance CSVD-H subgroup $\left(\mathrm{X}^{2}=5.239, \mathrm{p}=0.022\right)$. The behavioural profile of these two subgroups was plotted alongside Non-CSVD controls (Figure 5). A one-way between-groups multivariate 
analysis of variance (MANCOVA) was performed to investigate group differences in objective and subjective measures of cognition. Three dependent variables were used: EF score, PS score, and SC score. The independent variable was group membership. Age, education and WMH lesion burden were also entered as covariates. The MANCOVA revealed group differences with respect to $E F(F[2,39]=9.296, p<0.001$, partial eta squared $=0.323), \operatorname{PS}(F[2,39]=4.374, p=$ 0.019 , partial eta squared $=0.183)$ and $\mathrm{SC}(\mathrm{F}[2,39]=4.453, \mathrm{p}=0.018$, partial eta squared $=$ 0.186). Notably, results were largely unchanged if WMH burden was dropped as a covariate. Pairwise comparisons between healthy controls and the CSVD subgroups across the three cognitive measures (with Bonferroni correction), revealed that compared to healthy (non-CSVD) controls, those within the CSVD-H subgroup scored significantly lower on PS ( $p=0.045$, Cohen's $d=0.87$ ) and reported greater subjective complaints ( $\mathrm{p}=0.016$, Cohen's $d=1.28$ ) despite no statistically significant difference in executive function. In contrast, the CSVD-L scored significantly worse than controls on EF ( $\mathrm{p}=0.028$, Cohen's $d=1.13$ ) with no significant differences with respect to subjective complaints or processing speed. Pairwise comparisons are summarized in Table 5. Pairwise comparisons between the CSVD subgroups are also reported for descriptive purposes and not discussed as these differences were used to define the groups by the cluster analysis.

Table 4: Characteristics of CSVD behavioural sub-clusters

\begin{tabular}{lcccccc} 
& \multicolumn{2}{c}{ Non-CSVD } & \multicolumn{2}{c}{ CSVD-H } & \multicolumn{2}{c}{ CSVD-L } \\
Measure & Mean/Count & SD & Mean/Count & SD & Mean/Count & SD \\
\hline Sample Size & 23 & & 12 & & 11 & \\
Age & 69 & 5 & 70 & 6 & 72 & 4 \\
Years of Education & 16.8 & 3.1 & 16.4 & 3.3 & 17.3 & 4 \\
\# of Vascular Risk Factors & 3 & 2 & 3 & 2 & 3 & 1 \\
Gender (M: F) & $9: 14$ & & $3: 9 *$ & & $8: 3$ & \\
WMH Lesion Burden (\% ICV) & 0.143 & 0.112 & .714 & 0.770 & 1.048 & 0.658 \\
Lacune Lesion Burden $\left(\mathrm{cm}^{3}\right)$ & 0.018 & 0.02 & 0.269 & 0.316 & 0.279 & 0.419 \\
\# of Lacunes & 0 & 1 & 2 & 2 & 3 & 2 \\
Geriatric Depression Scale & 3.00 & 2.74 & 7.83 & 6.79 & 5.73 & 4.0 \\
\hline \hline
\end{tabular}

SD: standard deviation.

Table 5: Pairwise comparison between Non-CSVD and CSVD behavioural sub-groups

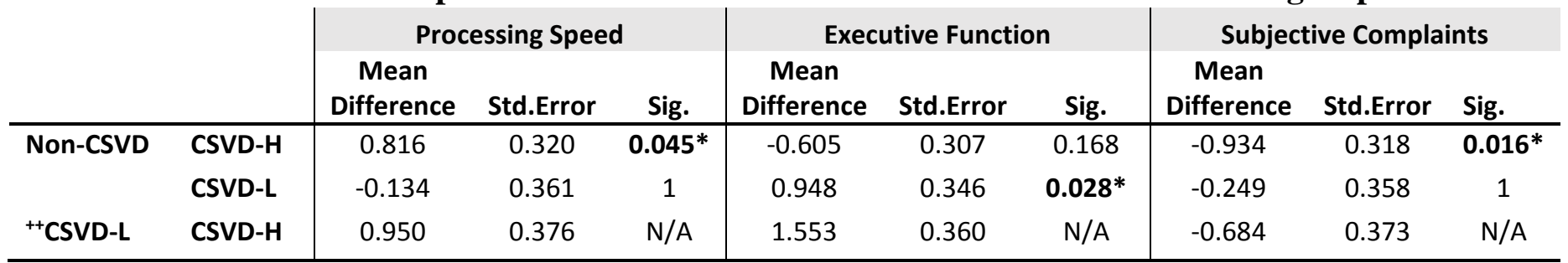


* Mean difference is significant at .05 level. Bonferroni adjustment for multiple comparisons applied. ${ }^{++}$For descriptive purposes only. Differences between CSVD subgroups were used to create the groups.

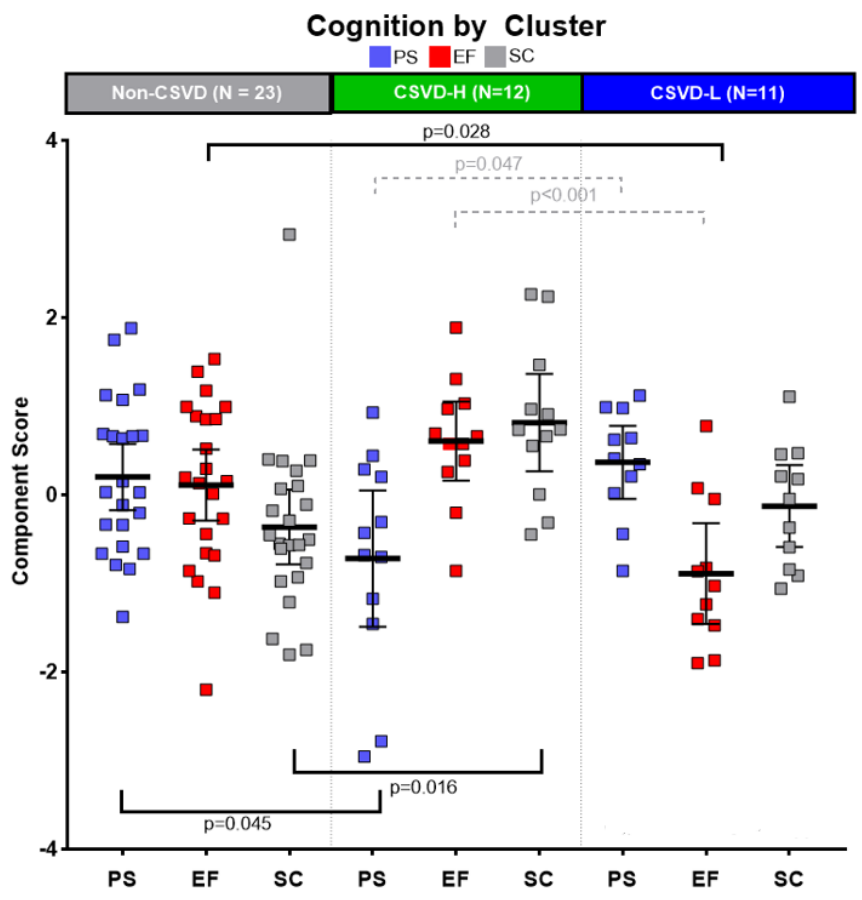

Figure 5. CSVD-high performers versus CSVD-low performers.

Dot plot comparing behavioural characteristics across all groups. Reported statistics represent Bonferroni adjusted pairwise comparisons between groups following adjustment for age, education and WMH lesion burden. Along the horizontal axis are the primary cognitive measures; processing speed component score (PS, purple), executive function component score (EF, red), and subjective complaints component score (SC, grey). The horizontal axis is subdivided into three sections for the three groups; Non-CSVD ( $=23)$, CSVD-H $(n=12)$, and CSVD-L ( $\mathrm{n}=11)$. Compared to Non-CSVD controls, the CSVD-H subgroup were significantly slower (PS, $\mathrm{p}=0.045)$ and reported greater subjective complaints $(\mathrm{SC}, \mathrm{p}=0.016)$ despite no statistically significant difference in executive function (EF). In contrast, the CSVD-L scored significantly worse than controls on $\mathrm{EF}(\mathrm{p}=0.028)$ with no significant differences with respect to subjective complaints (SC) or processing speed (PS).

\subsection{Discussion}

Half of the community dwelling older adults with vascular risk factors recruited for this study were found to have significant white matter hyperintensities, consistent with CSVD. This highlights how common white matter hyperintensities are in the general population, particularly among those with vascular risk factors. We sought to investigate the relationship between WMH lesion burden and objective and subjective measures of executive function, and to identify the pattern of deficits that distinguish older adults with and without CSVD. 


\subsection{Subjective complaints differentiated older adults with and without CSVD}

When older adults were divided into those with and without CSVD (WMH burden $>\sim 10 \mathrm{~cm}^{3}$ ), subjective complaints, but not objective test findings, significantly distinguished the two groups after adjusting for age and education. One explanation for this finding is that neuropsychological tests may not be sufficiently sensitive to detect executive dysfunction in high functioning older adults with only mild to moderate damage (Benedictus et al., 2015; Burgess et al., 2006). Impairments in executive functioning in such individuals may only become evident when performing complex real-world tasks as they may be able to compensate up to a certain level of difficulty by drawing on cognitive reserve and/or utilizing compensatory strategies. In patients with cerebrovascular disease, greater self-report of cognitive difficulties at baseline predicted poorer cognitive performance at 12-month follow-up (Haley et al., 2009). The value of tracking subjective cognitive decline in clinical populations was also recently highlighted by Vogel et al. (2017) who reported that among patients with $\beta$-amyloid pathology the rate of cognitive decline was most steep for those reporting the greatest subjective cognitive decline. In that study, subjective cognitive decline also predicted a faster decline in working memory independent of $\beta$ amyloid burden (see also Buckley et al. (2017). Overall these findings support the notion that subjective complaints may provide clinicians and researchers additional information regarding the cognitive effects of white matter lesion burden that may be missed by traditional cognitive testing.

\subsection{Inter-individual variability in CSVD may relate to compensation}

The fact that our objective test battery did not distinguish older adults with and without CSVD was remarkable given that it contained state-of-the-art clinical measures of executive function, attention, working memory, and simulated real life function. Although it was not sensitive to CSVD across all participants, we noted high heterogeneity within the CSVD population. Inspection of the density plots of neuropsychological test performance revealed a bimodal distribution with respect to executive function. This was supported by hierarchical cluster analysis, which revealed two distinct behavioural subgroups within our CSVD sample that differed with respect to executive function and processing speed, despite being similar with respect to age, education, and most importantly, WMH burden. This suggests that cognition in older adults with mild to moderate CSVD may be influenced by factors that cannot be readily captured by traditional clinical neuroimaging tools. That is, simply looking at total lesion burden 
may not provide an accurate reflection of cognitive status. One possibility is that inter-individual variability in CSVD may relate to differences in cognitive reserve and ability to compensate in response to age-related white matter changes, possibly accounting for previous findings of no significant relationship between EF and CSVD (Burns et al (2005).

The Scaffolding Theory of Aging and Cognition (STAC Model) (Reuter-Lorenz and Park, 2014) proposes that the adverse effects of neural decline may be attenuated through reorganizing/creating alternative neural circuits (compensatory scaffolds). These additional neural circuits provide the computational support needed to preserve cognitive function in the face of neurofunctional decline, albeit at the cost of efficiency. That is, there is a trade-off between performance and efficiency. Consistent with this idea, those in the higher performing CSVD group (CSVD-H) demonstrate preserved executive function and lower speed of processing despite reporting greater subjective complaints (relative to controls). In this group, one can argue that there has been a compensatory trade off between speed and accuracy resulting in preserved executive function but slower speed of processing. These individuals however still report high subjective complaints as they are still able to perceive that they are not as fast as before. However, not all individuals with CSVD are able to adjust. The poorer performing CSVD group (CSVD-L) demonstrate preserved speed of processing (relative to controls) despite relatively low executive function, suggesting failed/absence of compensation, with less subjective awareness of their deficits (Brookes et al., 2014).

\subsection{Limitations}

As the study is observational, conclusions regarding causality cannot be inferred. Despite a starting sample size of 46 and having equal numbers of older adults with and without CSVD, subgroup analysis of the CSVD sample yielded groups of only 12 and 11, which limits the generalizability of findings. Also, as our power calculations were based on effect sizes taken from a meta-analysis reporting a very large effect size (Vasquez et al., 2015), the power of our study is limited to the detection of effects beyond the range of what is conventionally considered to be a large effect. Furthermore, as large effects are often found in small samples due to overfitting, these findings would need to be replicated in an independent, preferably larger sample. Another potential limitation is the use of self-reported vascular risk factors, which tend to be under-reported in older adults (Dey et al., 2015). To mitigate this risk, we cross-checked their risk factors against participants' medications. Although participants were required to have 
at least one vascular risk factor to meet inclusion criteria, they reported an average of two more risk factors, suggesting that under-reporting was unlikely. Because recruitment was dependent on participants calling in response to ads or being registered within the Rotman Research Institute's participant directory, the study may be susceptible to recruitment bias which again limits the generalizability of findings. APOE 4 status was not screened for in this study. It is possible that some of the cognitive effects observed in this study may be partially related to the accumulation of beta-amyloid pathology (Vogel et al., 2017).

Those with CSVD reported more depressive symptoms relative to those without CSVD. The relationship between depression and cognitive deficits is well established (Rock et al., 2014) and could represent a possible confound to the extent that depressive symptoms could be associated with test scores. Although none of our participants met criteria for a diagnosis of depression, we examined the relationship of GDS scores to the component scores from our neuropsychological battery. There were no significant correlations between GDS scores and EF $(r=-0.042, p=$ $0.784)$ or PS scores $(r=-0.146, p=0.339)$, nor did GDS scores significantly differ between the two CSVD subgroups. The only statistically significant relationship observed was between GDS scores and subjective complaints $(\mathrm{r}=0.62, \mathrm{n}=45, \mathrm{p}<0.001)$, likely accounted for by overlapping questions within the GDS that pertain to memory and concentration (Adams et al., 2004; Hohman et al., 2011). It is possible that the presence of CSVD may predispose individuals to developing depressive symptoms (Sloten et al (2015), possibly through disruption of brain structures involved in mood regulation (Taylor et al., 2013). Regardless, the presence of concurrent depressive symptoms does not invalidate our participants subjective experience of cognitive decline. Indeed, participant self-report was corroborated by the collection of paired assessments from a close relative. With respect to sex-related differences, while there were no significant differences in $\mathrm{EF}[(\mathrm{t} 44)=-0.816, \mathrm{p}=0.419)], \mathrm{PS}[(\mathrm{t} 44)=1.564, \mathrm{p}=0.125)]$ or SC $[\mathrm{t}(44)=0.691, \mathrm{p}=0.493]$ scores between males and females, females were over-represented in the CSVD-H group. These findings are contrary to what is typically reported in the acquired brain injury literature wherein functional outcomes and quality of life after a stroke or mild TBI are consistently poorer in women (Bazarian et al., 2010; Reeves et al., 2008). Further research into sex-differences in cognition and cognitive reserve in older adults with CSVD is recommended. Another limitation in our study relates to the definition of CSVD used. At present there is no clear consensus in the literature with respect to what amount of lesion burden equates to 
clinically significant CSVD. For this study we utilized a quantitative cut-off of lesion burden as reported by DeCarli et al. (1995) as this was the most objective and widely accepted definition we identified in the literature at the outset of our study that continues to be used (Chao et al., 2013; Svärd et al., 2017; White et al., 2013). More recently, several groups have started using an ordinal SVD score of 0-4, representing the total MRI load of CSVD by counting the presence of 4 MRI features (WMHs, lacunes, microbleeds, and enlarged perivascular spaces) (Staals et al., 2015). The problem with this score however is that is overly reliant on visual scales that are troubled by issues such as poor sensitivity to group differences, high inter-subject variability and significant ceiling/floor effects. These issues limit the utility of this measure. Indeed, Staals et al. (2015) found no association between total SVD load and processing speed and memory. As such we have avoided such scales. With that said, a $0.5 \% \mathrm{WMH}$ volumetric threshold roughly corresponds to a Fazekas score of 1, while volumes over 1\% of intracranial volume correspond to Fazekas scores of 2 and above (Svärd et al., 2017).

Next, although as WMH volume was extracted from 3mm FLAIR slices (as per current imaging guidelines), it is plausible that smaller WMH volumes may have been missed in our study. Any sub $3 \mathrm{~mm}$ lesions missed likely would have had little impact on the interpretation of our results.

Finally, in this study, a global measure of WMH burden was utilized, whereas regional differences in the distribution of lesion burden may have provided more information and may have shared a stronger relationship with measures of cognition. For example, lower cognitive speed and flexibility is associated with WMH burden in the superior corona radiata (Birdsill et al., 2014). That said, when we repeated our analyses using regional WMH volumes, no significant correlations with any of the cognitive measures was found. This may relate to the mild degree of severity in this community sample of individuals with risk factors (but not diagnosed CSVD), where regional effects may not be sufficient to account for variance relative to the diffuse effects of global lesion burden.

\subsection{Conclusion}

This study aimed to investigate the relationship between ischemic lesion burden and objective and subjective measures of cognitive function in a sample of older community-dwelling adults with vascular risk factors. Half of our recruited community sample were found to have neuroimaging evidence of CSVD, highlighting the commonality of such changes that should be 
considered when interpreting findings in older adults. We found distinct behavioural subgroups that were matched with respect to age, education and WMH burden - separating those who could compensate (at the cost of reduced speed of processing) versus those who could not. This may provide some explanation for the large behavioural heterogeneity often observed in studies of age-related white matter changes. Finally, subjective complaints but not objective test scores differentiated those with and without CSVD, supporting the use of subjective measures of cognitive impairment. Such measures can supplement objective testing by detecting subtle effects of pathology that are difficult to reliably measure via laboratory testing and that may be masked by individual differences in cognitive reserve and compensation. These findings encourage future investigation into functional brain changes associated with white matter hyperintensities to provide insights into the contribution of brain reserve and compensation to individual differences in cognitive abilities as humans age. Ultimately, a better understanding of the mechanisms contributing to cognitive changes, whether objectively or subjectively measured, may have implications for guiding the development of novel and more targeted assessment and intervention strategies for older adults with mild to moderate cerebral small vessel disease.

\section{Acknowledgements}

This research was supported by a Canadian Institute for Health Research (CIHR) MD/ Ph.D. Studentship award to Ayan Dey, a CIHR grant \#MOP-202037 awarded to B. Levine, and financial support from the Heart and Stroke Foundation Canadian Partnership for Stroke Recovery. Authors would also like to acknowledge Dr. Esopenko for consultation on the project and research assistants: Wayne Khuu and Nicola De Souza for their technical support as well as Priya Kumar, Laura Oliva, Alissa Papadopoulos and Laryssa Levesque for assistance in participant recruitment and testing.

\section{Declarations of Interest}

None 


\section{References}

Adams, K.B., Matto, H.C., Sanders, S., 2004. Confirmatory factor analysis of the geriatric depression scale. The Gerontologist 44(6), 818-826.

Bazarian, J.J., Blyth, B., Mookerjee, S., He, H., McDermott, M.P., 2010. Sex differences in outcome after mild traumatic brain injury. Journal of neurotrauma 27(3), 527-539.

Benedict, R.-H.B., Schretlen, D., Groninger, L., Brandt, J., 1998. Hopkins Verbal Learning Test--Revised: Normative data and analysis of inter-form and test-retest reliability. Clinical Neuropsychologist 12(1), 43-55.

Benedictus, M.R., van Harten, A.C., Leeuwis, A.E., Koene, T., Scheltens, P., Barkhof, F., Prins, N.D., van der Flier, W.M., 2015. White Matter Hyperintensities Relate to Clinical Progression in Subjective Cognitive Decline. Stroke 46(9), 2661-2664.

Birdsill, A.C., Koscik, R.L., Jonaitis, E.M., Johnson, S.C., Okonkwo, O.C., Hermann, B.P., Larue, A., Sager, M.A., Bendlin, B.B., 2014. Regional white matter hyperintensities: aging, Alzheimer's disease risk, and cognitive function. Neurobiology of aging 35(4), 769-776.

Broadbent, D.E., Cooper, P.F., FitzGerald, P., Parkes, K.R., 1982. The Cognitive Failures Questionnaire (CFQ) and its correlates. Br J Clin Psychol 21(Pt 1), 1-16.

Brookes, R.L., Herbert, V., Paul, S., Hannesdottir, K., Markus, H.S., Morris, R.G., 2014. Executive dysfunction, awareness deficits and quality of life in patients with cerebral small vessel disease: a structural equation model. Neuropsychology 28(2), 247-253.

Buckley, R.F., Hanseeuw, B., Schultz, A.P., Vannini, P., Aghjayan, S.L., Properzi, M.J., Jackson, J.D., Mormino, E.C., Rentz, D.M., Sperling, R.A., Johnson, K.A., Amariglio, R.E., 2017. Region-Specific Association of Subjective Cognitive Decline With Tauopathy Independent of Global beta-Amyloid Burden. JAMA Neurol 74(12), 1455-1463.

Burgess, P.W., Alderman, N., Forbes, C., Costello, A., Coates, L.M., Dawson, D.R., Anderson, N.D., Gilbert, S.J., Dumontheil, I., Channon, S., 2006. The case for the development and use of "ecologically valid" measures of executive function in experimental and clinical neuropsychology. J Int Neuropsychol Soc 12(2), 194-209.

Burns, J.M., Church, J.A., Johnson, D.K., Xiong, C., Marcus, D., Fotenos, A.F., Snyder, A.Z., Morris, J.C., Buckner, R.L., 2005. White matter lesions are prevalent but differentially related with cognition in aging and early Alzheimer disease. Archives of neurology 62(12), 1870-1876.

Chao, L.L., Decarli, C., Kriger, S., Truran, D., Zhang, Y., Laxamana, J., Villeneuve, S., Jagust, W.J., Sanossian, N., Mack, W.J., Chui, H.C., Weiner, M.W., 2013. Associations between white matter hyperintensities and beta amyloid on integrity of projection, association, and limbic fiber tracts measured with diffusion tensor MRI. PloS one 8(6), e65175.

Dade, L.A., Gao, F.Q., Kovacevic, N., Roy, P., Rockel, C., O'Toole, C.M., Lobaugh, N.J., Feinstein, A., Levine, B., Black, S.E., 2004. Semiautomatic brain region extraction: a method of parcellating brain regions from structural magnetic resonance images. Neuroimage 22(4), 1492-1502.

DeCarli, C., Murphy, D.G., Tranh, M., Grady, C.L., Haxby, J.V., Gillette, J.A., Salerno, J.A., Gonzales-Aviles, A., Horwitz, B., Rapoport, S.I., et al., 1995. The effect of white matter hyperintensity volume on brain structure, cognitive performance, and cerebral metabolism of glucose in 51 healthy adults. Neurology 45(11), 2077-2084.

Delis, D.C., Kaplan, E., Kramer, J.H., 2001. Delis-Kaplan executive function system (D-KEFS). Psychological Corporation.

Dey, A.K., Alyass, A., Muir, R.T., Black, S.E., Swartz, R.H., Murray, B.J., Boulos, M.I., 2015. Validity of SelfReport of Cardiovascular Risk Factors in a Population at High Risk for Stroke. Journal of stroke and cerebrovascular diseases : the official journal of National Stroke Association 24(12), 28602865. 
Fazekas, F., Chawluk, J.B., Alavi, A., Hurtig, H.I., Zimmerman, R.A., 1987. MR signal abnormalities at 1.5 T in Alzheimer's dementia and normal aging. AJR. American journal of roentgenology 149(2), 351356.

Gibson, E., Gao, F., Black, S.E., Lobaugh, N.J., 2010. Automatic segmentation of white matter hyperintensities in the elderly using FLAIR images at 3T. Journal of Magnetic Resonance Imaging 31(6), 1311-1322.

Gouw, A.A., Seewann, A., van der Flier, W.M., Barkhof, F., Rozemuller, A.M., Scheltens, P., Geurts, J.J., 2011. Heterogeneity of small vessel disease: a systematic review of MRI and histopathology correlations. J Neurol Neurosurg Psychiatry 82(2), 126-135.

Haley, A.P., Hoth, K.F., Gunstad, J., Paul, R.H., Jefferson, A.L., Tate, D.F., Ono, M., Jerskey, B.A., Poppas, A., Sweet, L.H., Cohen, R.A., 2009. Subjective Cognitive Complaints Relate to White Matter Hyperintensities and Future Cognitive Decline in Patients with Cardiovascular Disease. The American journal of geriatric psychiatry : official journal of the American Association for Geriatric Psychiatry 17(11), 976.

Hohman, T.J., Beason-Held, L.L., Resnick, S.M., 2011. Cognitive complaints, depressive symptoms, and cognitive impairment: are they related? J Am Geriatr Soc 59(10), 1908-1912.

Jellinger, K.A., 2013. Pathology and pathogenesis of vascular cognitive impairment-a critical update. Front Aging Neurosci 5, 17.

Khan, U., Porteous, L., Hassan, A., Markus, H.S., 2007. Risk factor profile of cerebral small vessel disease and its subtypes. Journal of Neurology, Neurosurgery, and Psychiatry 78(7), 702-706.

Kovacevic, N., Lobaugh, N.J., Bronskill, M.J., Levine, B., Feinstein, A., Black, S.E., 2002. A robust method for extraction and automatic segmentation of brain images. Neuroimage 17(3), 1087-1100.

Lawrence, A.J., Brookes, R.L., Zeestraten, E.A., Barrick, T.R., Morris, R.G., Markus, H.S., 2015. Pattern and Rate of Cognitive Decline in Cerebral Small Vessel Disease: A Prospective Study. PloS one 10(8), e0135523.

Longstreth, W.T., Jr., Dulberg, C., Manolio, T.A., Lewis, M.R., Beauchamp, N.J., Jr., O'Leary, D., Carr, J., Furberg, C.D., 2002. Incidence, manifestations, and predictors of brain infarcts defined by serial cranial magnetic resonance imaging in the elderly: the Cardiovascular Health Study. Stroke 33(10), 2376-2382.

Manly, T., Hawkins, K., Evans, J., Woldt, K., Robertson, I.H., 2002. Rehabilitation of executive function: facilitation of effective goal management on complex tasks using periodic auditory alerts. Neuropsychologia 40(3), 271-281.

Martins, R., Joanette, Y., Monchi, O., 2015. The implications of age-related neurofunctional compensatory mechanisms in executive function and language processing including the new Temporal Hypothesis for Compensation. Frontiers in Human Neuroscience 9(221).

Miyake, A., Friedman, N.P., Emerson, M.J., Witzki, A.H., Howerter, A., Wager, T.D., 2000. The unity and diversity of executive functions and their contributions to complex "Frontal Lobe" tasks: a latent variable analysis. Cogn Psychol 41(1), 49-100.

Molad, J., Kliper, E., Korczyn, A.D., Ben Assayag, E., Ben Bashat, D., Shenhar-Tsarfaty, S., Aizenstein, O., Shopin, L., Bornstein, N.M., Auriel, E., 2017. Only White Matter Hyperintensities Predicts PostStroke Cognitive Performances Among Cerebral Small Vessel Disease Markers: Results from the TABASCO Study. J Alzheimers Dis 56(4), 1293-1299.

Petrides, M., Milner, B., 1982. Deficits on subject-ordered tasks after frontal- and temporal-lobe lesions in man. Neuropsychologia 20, 249-262.

Prins, N.D., van Dijk, E.J., den Heijer, T., Vermeer, S.E., Jolles, J., Koudstaal, P.J., Hofman, A., Breteler, M.M., 2005. Cerebral small-vessel disease and decline in information processing speed, executive function and memory. Brain : a journal of neurology 128(Pt 9), 2034-2041. 
Ramirez, J., Gibson, E., Quddus, A., Lobaugh, N.J., Feinstein, A., Levine, B., Scott, C.J., Levy-Cooperman, N., Gao, F.Q., Black, S.E., 2011. Lesion Explorer: a comprehensive segmentation and parcellation package to obtain regional volumetrics for subcortical hyperintensities and intracranial tissue. Neuroimage 54(2), 963-973.

Reeves, M.J., Bushnell, C.D., Howard, G., Gargano, J.W., Duncan, P.W., Lynch, G., Khatiwoda, A., Lisabeth, L., 2008. Sex differences in stroke: epidemiology, clinical presentation, medical care, and outcomes. Lancet neurology 7(10), 915-926.

Reuter-Lorenz, P.A., Park, D.C., 2014. How Does it STAC Up? Revisiting the Scaffolding Theory of Aging and Cognition. Neuropsychology Review 24(3), 355-370.

Rincon, F., Wright, C., 2014. Current pathophysiological concepts in cerebral small vessel disease. Frontiers in Aging Neuroscience 6(24).

Robertson, I.H., Ward, T., Ridgeway, V., Nimmo-Smith, I., 1996. The structure of normal human attention: The Test of Everyday Attention. J. Int. Neuropsychol. Soc. 2(6), 525-534.

Rock, P.L., Roiser, J.P., Riedel, W.J., Blackwell, A.D., 2014. Cognitive impairment in depression: a systematic review and meta-analysis. Psychological medicine 44(10), 2029-2040.

Rost, N.S., Sadaghiani, S., Biffi, A., Fitzpatrick, K.M., Cloonan, L., Rosand, J., Shibata, D.K., Mosley, T.H., Jr., 2014. Setting a gold standard for quantification of leukoaraiosis burden in patients with ischemic stroke: the Atherosclerosis Risk in Communities Study. Journal of neuroscience methods 221, 196-201.

Sachdev, P., Kalaria, R., O'Brien, J., Skoog, I., Alladi, S., Black, S.E., Blacker, D., Blazer, D.G., Chen, C., Chui, H., Ganguli, M., Jellinger, K., Jeste, D.V., Pasquier, F., Paulsen, J., Prins, N., Rockwood, K., Roman, G., Scheltens, P., 2014. Diagnostic criteria for vascular cognitive disorders: a VASCOG statement. Alzheimer disease and associated disorders 28(3), 206-218.

Smith, A., 1978. Symbol Digit Modalities Test. Western Psychological Services, Los Angeles.

Spreen, O., Strauss, E., 1998. A compendium of neuropsychological tests: Administration, norms, and commentary. Oxford University Press, New York.

Staals, J., Booth, T., Morris, Z., Bastin, M.E., Gow, A.J., Corley, J., Redmond, P., Starr, J.M., Deary, I.J., Wardlaw, J.M., 2015. Total MRI load of cerebral small vessel disease and cognitive ability in older people. Neurobiology of aging 36(10), 2806-2811.

Svärd, D., Nilsson, M., Lampinen, B., Lätt, J., Sundgren, P.C., Stomrud, E., Minthon, L., Hansson, O., van Westen, D., 2017. The effect of white matter hyperintensities on statistical analysis of diffusion tensor imaging in cognitively healthy elderly and prodromal Alzheimer's disease. PloS one 12(9), e0185239.

Taylor, W.D., Aizenstein, H.J., Alexopoulos, G.S., 2013. The vascular depression hypothesis: mechanisms linking vascular disease with depression. Molecular psychiatry 18(9), 963-974.

Uiterwijk, R., van Oostenbrugge, R.J., Huijts, M., De Leeuw, P.W., Kroon, A.A., Staals, J., 2016. Total Cerebral Small Vessel Disease MRI Score Is Associated with Cognitive Decline in Executive Function in Patients with Hypertension. Front Aging Neurosci 8, 301.

van Dijk, E.J., Prins, N.D., Vrooman, H.A., Hofman, A., Koudstaal, P.J., Breteler, M.M., 2008. Progression of cerebral small vessel disease in relation to risk factors and cognitive consequences: Rotterdam Scan study. Stroke 39(10), 2712-2719.

van Sloten, T.T., Sigurdsson, S., van Buchem, M.A., Phillips, C.L., Jonsson, P.V., Ding, J., Schram, M.T., Harris, T.B., Gudnason, V., Launer, L.J., 2015. Cerebral Small Vessel Disease and Association With Higher Incidence of Depressive Symptoms in a General Elderly Population: The AGES-Reykjavik Study. The American journal of psychiatry 172(6), 570-578.

Vasquez, B.P., Zakzanis, K.K., 2015. The neuropsychological profile of vascular cognitive impairment not demented: a meta-analysis. Journal of neuropsychology 9(1), 109-136. 
Vogel, J.W., Varga Dolezalova, M., La Joie, R., Marks, S.M., Schwimmer, H.D., Landau, S.M., Jagust, W.J., 2017. Subjective cognitive decline and beta-amyloid burden predict cognitive change in healthy elderly. Neurology 89(19), 2002-2009.

Wardlaw, J.M., Smith, C., Dichgans, M., 2013a. Mechanisms of sporadic cerebral small vessel disease: insights from neuroimaging. Lancet Neurol 12(5), 483-497.

Wardlaw, J.M., Smith, E.E., Biessels, G.J., Cordonnier, C., Fazekas, F., Frayne, R., Lindley, R.I., O'Brien, J.T., Barkhof, F., Benavente, O.R., Black, S.E., Brayne, C., Breteler, M., Chabriat, H., Decarli, C., de Leeuw, F.E., Doubal, F., Duering, M., Fox, N.C., Greenberg, S., Hachinski, V., Kilimann, I., Mok, V., Oostenbrugge, R., Pantoni, L., Speck, O., Stephan, B.C., Teipel, S., Viswanathan, A., Werring, D., Chen, C., Smith, C., van Buchem, M., Norrving, B., Gorelick, P.B., Dichgans, M., 2013b. Neuroimaging standards for research into small vessel disease and its contribution to ageing and neurodegeneration. Lancet Neurol 12(8), 822-838.

Wen, W., Sachdev, P.S., Li, J.J., Chen, X., Anstey, K.J., 2009. White matter hyperintensities in the forties: Their prevalence and topography in an epidemiological sample aged 44-48. Human brain mapping 30(4), 1155-1167.

White, W.B., Marfatia, R., Schmidt, J., Wakefield, D.B., Kaplan, R.F., Bohannon, R.W., Hall, C.B., Guttmann, C.R., Moscufo, N., Fellows, D., Wolfson, L., 2013. INtensive versus standard ambulatory blood pressure lowering to prevent functional DeclINe in the ElderlY (INFINITY). American heart journal 165(3), 258-265 e251.

Wilson, B.A., Alderman, N., Burgess, P.W., Emslie, H., Evans, J.J., 1996. Behavioral Assessment of the Dysexecutive Syndrome. Thames Valley Test Company, Bury St. Edmunds.

Yesavage, J.A., Brink, T.L., Rose, T.L., Lum, O., Huang, V., Adey, M., Leirer, V.O., 1982. Development and validation of a geriatric depression screening scale: a preliminary report. Journal of psychiatric research 17(1), 37-49.

Yushkevich, P.A., Piven, J., Hazlett, H.C., Smith, R.G., Ho, S., Gee, J.C., Gerig, G., 2006. User-guided 3D active contour segmentation of anatomical structures: significantly improved efficiency and reliability. Neuroimage 31(3), 1116-1128.

Zheng, J.J.J., Lord, S.R., Close, J.C.T., Sachdev, P.S., Wen, W., Brodaty, H., Delbaere, K., 2012. Brain White Matter Hyperintensities, Executive Dysfunction, Instability, and Falls in Older People: A Prospective Cohort Study. The Journals of Gerontology: Series A 67(10), 1085-1091. 